(3) M. Yamada : Bull. Agricult. Chem, Soc, Japan, 3, 76 80 (1927); Chem. Zent., II, 2479 (1928).

A. Trillat et Sauton: Compt, rend., 146, 996 9 (1908).

(4) M. Yamada: Bull. Agricult. Chem. Soc. Japan, 4, 18 21 (1928); Chem. Zent., II, 585 (1929); Rull. Agricult. Chem. Suc. Japan, 4, 89 91 (1928); also see

S. Akabori: Proc. Imp. Acad. Tokyo, 3, 672 4 (1927); Chem. Zent., I, 1757 (1928).

\title{
On the Alcoholic Fermentation of the Amino-acid. Part III.
}

$\alpha$-amino-n-butyric acid.

\author{
By \\ Masakazu YAMADA. \\ (Imp. Brew. Experimental Station, Takinogawa machi near Tokyo.)
}

(Received June 7, 19.32.)

Abderhalden, Chang and Wurm obtained $l$-isomeride as the decomposition product by yeast from $r$-amino- $n$-butyric acid and supposed that $d$-component was the naturally occurring isomeride. But then they did not pay attention to the volatile products. According to Ehrlich's view n-propylalcohol ought to be produced from the amino acid.

The author's experiment showed that the fusel oil fraction produced by sake yeast in the modified Hayduck solution with this amino acid in place of asparagine consisted mainly of an amyl alcohol which boiled at about 123 $8^{\circ}$. From the rotatory power of the alcohol and the properties of two derivatives, this was identified as active amyl alcohol.

\section{Experimental.}

$d-l$ - $\alpha$-amino-n-butyric acid was prepared from $n$-propyl alcohol merck.

Medium: Cane sugar $100 \mathrm{~g}$., amino acid $3 \mathrm{~g}$., Hayduck mineral solution 20 c.c., water 980 c.c.

1 to 2.51 . of culture medium in a 2 to 51 . flask was sterilized in ordinary way and then saké yeast was added-23 g. as dry yeast for total 13.2 1. of the medium. After 11 days at $25 \sim 9^{\circ}$ the fermentation was over and the fermented liquid contained no sugar. The distillation and the treatment of the residue are the same as the case of alanine (see part II).

\begin{tabular}{|c|c|c|c|}
\hline & Alcohol & Total acid & Fusel oil \\
\hline $\begin{array}{l}\text { Fermentation products } \\
\text { Iield of alcohol }\end{array}$ & $\begin{array}{c}4.4 \% \\
632 \text { c.c. }(93 \%)\end{array}$ & $\begin{array}{c}0.1416 \% \\
24(90 \%) \\
\text { (fusel oil } 0.768 \mathrm{~g} \text {.) }\end{array}$ & $\begin{array}{c}\text { 0.07\% } \\
\left.\text { oil (B. P. over } 100^{\circ}\right) \\
7.3 \text { g. }\end{array}$ \\
\hline
\end{tabular}


Fractionation of the oil.

\begin{tabular}{l|c|c||c|c|c}
\hline & B. P. & Yield (g.) & & B. P. & Yield (g.) \\
\hline I & $100 \sim 107^{\circ}$ & 0.2 & V & $126-127.5^{\circ}$ & 1.7 \\
II & $107 \sim 116^{\circ}$ & 0.3 & VI & $127.5 \sim 128.5$ & 2.0 \\
III & $116 \sim 123^{\circ}$ & 0.1 & VII & residue & 0.5 \\
IV & $123 \sim 126^{\circ}$ & 1.7 & & & \\
\hline
\end{tabular}

Identification of the fractions.

The following derivatives of alcohol were prepared.

Phenyl carbamate :-

\begin{tabular}{c|c|c|c|c|c|c}
\hline \hline \multirow{2}{*}{ Fract. } & \multirow{2}{*}{ M. P. } & \multicolumn{5}{|c}{ Analysis } \\
\cline { 3 - 7 } & Subst. (g.) & $\mathrm{N}$ (c.c.) & $\mathrm{T}$. & $\mathrm{P} .(\mathrm{m}, \mathrm{m})$. & $\mathrm{N}(\%)$ fuund \\
\hline Act. amyl alcohol & $32.5^{\circ}$ & 0.0715 & 4.2 & $15^{\circ}$ & 765 & $\begin{array}{c}6.93 \\
6.76 \\
\left(\mathrm{C}_{12} \mathrm{II}_{12} \mathrm{O}_{2} \mathrm{~N}\right)\end{array}$ \\
\hline
\end{tabular}

3-5-dinitrobenzoate :-

\begin{tabular}{|c|c|c|c|c|c|c|}
\hline \multirow{2}{*}{ Fract. } & \multirow{2}{*}{ M. P. } & \multicolumn{5}{|c|}{ Analysis } \\
\hline & & Subst. (g.) & $N(c . c)$. & $\mathrm{T}$. & $\mathrm{P},(\mathrm{m}, \mathrm{m})$. & $N(0, o)$ found \\
\hline IV & $80^{\circ}$ & 0.0564 & 4.7 & $15^{\circ}$ & 768.3 & 9.89 \\
\hline $\mathrm{V}$ & 81.5 & 0.0666 & 5.7 & 15 & 760.5 & 10.05 \\
\hline Amyl alcchol & & & & & & $\begin{array}{c}9.93 \\
\left(\mathrm{C}_{12} \mathrm{H}_{14} \mathrm{O}_{6} \mathrm{~N}_{2}\right)\end{array}$ \\
\hline
\end{tabular}

Rotation of alcohol (mixture of IV, V, VI).

$$
\begin{aligned}
& {[\alpha]_{\mathrm{D}}^{93^{\circ}}=-5.55^{\circ} \quad r=-3.62^{\circ} \quad l=2.2 \mathrm{dm} . \quad c=29.64 \%} \\
& {[\alpha]_{\mathrm{D}}^{200}=-5.90^{\circ} \quad \text { (methyl ethyl carbin carbinol). }}
\end{aligned}
$$

Amino acid recovered from the distilling residue was $7.6 \mathrm{~g}$. and its rotation in $20 \% \mathrm{HCl}$ solution was following.

$$
[\alpha]_{\mathrm{D}}^{\circ \circ}=-1.14^{\circ} \quad r=-0.16^{\circ} \quad l=2.2 \mathrm{dm} . \quad c=6.37 \%
$$

